\title{
Chemical Shift Anisotropy in Powdered Solids Studied by 2D FT NMR with Flipping of the Spinning Axis
}

\author{
ad BaX, Nikolaus M. Szeverenyi, and Gary E. Maciel* \\ Department of Chemistry, Colorado State University, Fort Collins, Colorado 80523
}

Received August 22, 1983

Information about chemical shift anisotropy is usually difficult to extract from the spectrum of a nonspinning powdered sample because of the usually extensive overlap of the powder patterns from the chemically different sites in the molecule. Several types of experiments have been proposed to facilitate the measurement of chemical shift anisotropy (1-9). All have the common feature that the sample is rotated about the magic-angle axis $(1-8)$ or an axis very close to the magic angle $(9)$, either by rapid sample spinning $(1-7,9)$ or by rotating the sample in three discrete steps $(8)$.

We propose a new two-dimensional approach for obtaining the anisotropy information. In this new experiment, the spinning axis of the sample is flipped from 90 to $54.7^{\circ}$ between the evolution and detection periods. The experiment appears to be widely applicable and has great promise for the study of complex samples.

The experimental scheme is set out in Fig. 1. Cross polarization of, in our case, ${ }^{13} \mathrm{C}$ nuclei is performed while the sample is spun about an axis that makes an angle of $90^{\circ}$ with the static magnetic field. It can be shown (10) that the powder anisotropy pattern that obtains under these conditions is reversed and collapsed to half the width of the static nonspinning case, but keeps the same shape. At the end of the evolution period $\left(t_{1}\right)$, the $x$ component of the transverse ${ }^{13} \mathrm{C}$ magnetization is stored along the $z$ axis, parallel to the static magnetic field, by means of a $90_{y}{ }^{\circ} \mathrm{C}$ pulse. The orientation of the spinning axis of the sample is then changed to the magic angle. The sample is spun fast compared with the width of the anisotropy patterns, so that spinning sidebands have negligible intensities. A final $90^{\circ}{ }^{13} \mathrm{C}$ pulse rotates the $z$-stored ${ }^{13} \mathrm{C}$ magnetization back into the transverse plane, where it precesses in the time domain, $t_{2}$, with the corresponding isotropic chemical shift frequencies.

Cycling of the phase of the first $90^{\circ}{ }^{13} \mathrm{C}$ pulse alternately along $+y$ and $-y$, together with adding and subtracting of the acquired data, is used to eliminate spurious signals. The detected isotropic spectrum, $S\left(t_{1}, F_{2}\right)$ obtained by Fourier transformation with respect to $t_{2}$, is modulated in amplitude with the frequencies existing during the evolution period, $t_{1}$. Hence, the powder anisotropy information and the isotropic chemical shifts will appear in the $F_{1}$ dimension. Because of the amplitude modulation, a pure $2 \mathrm{D}$ absorption spectrum can be obtained by calculating the cosine Fourier transform, $S^{c c}\left(F_{1}, F_{2}\right)(11,12)$.

* To whom correspondence should be addressed. 


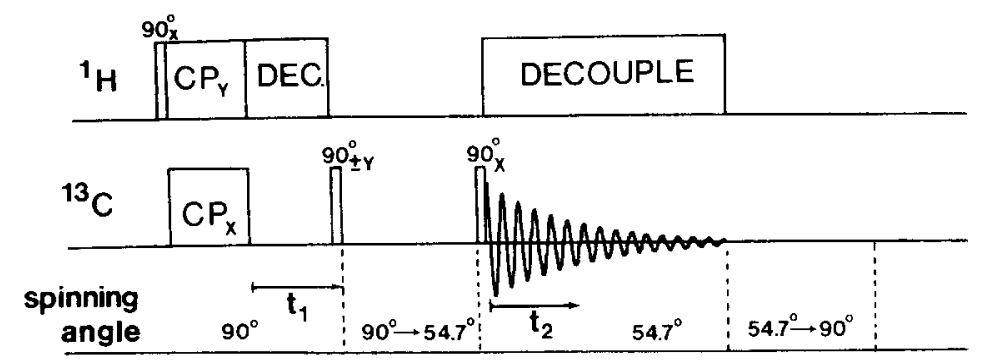

FIG. 1. Schematic representation of the two-dimensional experiment for mapping out the CSA pattern in the $F_{1}$ dimension. The angle between the sample spinning axis and the static magnetic field is changed from 90 to $54.7^{\circ}$ between the evolution and detection periods. In the present case a sample spinning at $2.5 \mathrm{kHz}$ undergoes this change of angle in $0.5 \mathrm{sec}$.

The method is demonstrated for a sample of polycrystalline p-dimethoxybenzene. Experiments were performed on a home-built spectrometer, equipped with a $2.35 \mathrm{~T}$ superconducting magnet and a Nicolet $293 \mathrm{~B}$ pulse programmer. The sample was spun at $2.3 \mathrm{kHz}$ in a turbine/air bearing spinning system obtained from Chemagnetics, Inc. (13). The orientation of the spinning axis was changed by means of a computercontrolled stepper motor from 90 to $54.7^{\circ}$ in a time less than 1 sec. Further details about the experimental setup will appear in a forthcoming publication. The length of the evolution period was incremented in 64 steps of $83 \mu \mathrm{sec}$ each, giving a spectral

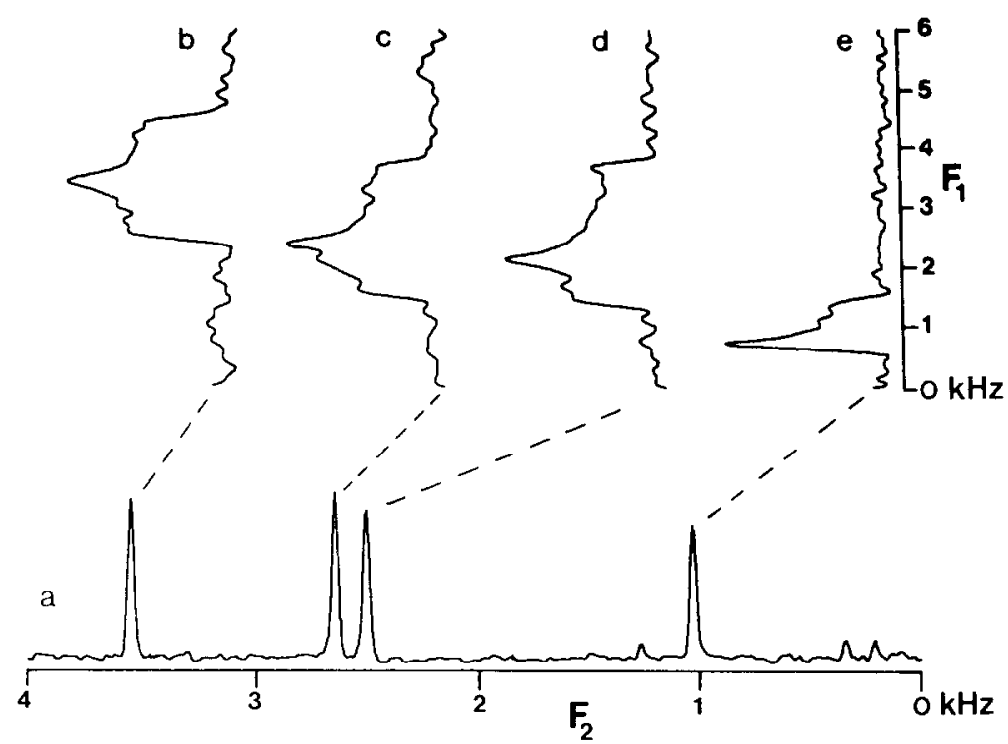

FIG. 2. (a) Phase-sensitive projection of the absorption-mode $2 \mathrm{D}$ spectrum of $p$-dimethoxybenzene, obtained with the sequence of Fig. 1, onto the $F_{2}$ axis, and (b-e) absorption mode cross sections through the $2 \mathrm{D}$ spectrum parallel to the $F_{1}$ axis, displaying the anisotropy patterns for the various sites. Because the ${ }^{13} \mathrm{C}$ transmitter frequency was placed at the high-shielding side of the spectrum, the anisotropy patterns have the conventional shape (not the mirror image). The patterns are half as wide as what would be obtained from the static, nonspinning case. 
TABLE 1

MEASURED COMPONENTS, $\sigma_{11}, \sigma_{22}$, AND $\sigma_{33}$, OF THE ${ }^{13} \mathrm{C}$ CHEMICAL
SHIFT SHIELDING TENSOR OF $p$-DIMETHOXYBENZENE GIVEN WITH
RESPECT TO THE CORRESPONDING ISOTROPIC
SHIELDING, $\sigma_{\mathbf{i}}($ in ppm)

width of $6.025 \mathrm{kHz}$ in the $F_{1}$ dimension, and 24 transients were recorded for each value of $t_{1}$. The total measuring time was approximately $2.5 \mathrm{hr}$. The ${ }^{13} \mathrm{C}$ transmitter frequency was placed at the high-shielding side of the ${ }^{13} \mathrm{C}$ spectrum.

Figure 2 shows the isotropic shift spectrum obtained from a phase-sensitive projection (14) of the 2D spectrum onto the $F_{2}$ axis, and absorption-mode cross sections parallel to the $F_{1}$ axis through the 2D spectrum taken at the various isotropic $F_{2}$ shift frequencies, displaying the individual anisotropy powder patterns. Values for the ${ }^{13} \mathrm{C}$ chemical shift tensor components measured from the patterns shown in Fig. 2 are given in Table 1 and are in good agreement with those given by Maricq and Waugh (I).

Our new experiment yields the anisotropy information in a very straightforward way with hardly any distortion. The sensitivity of the experiment is rather good; but it is, of course, lower than that of the conventional CP/MAS experiment carried out in the same amount of time. Sensitivity decreases if very high resolution in the anisotropy pattern is needed, because in this case a long sampling time in the $t_{1}$ dimension is required and for long $t_{1}$ values only very little signal is acquired. The experiment is not very sensitive to the adjustment of experimental parameters such as pulse width, etc., and requires only a stable spinning device that remains stable when the orientation of the spinning axis is changed rapidly during the course of the experiment.

\section{ACKNOWLEDGMENTS}

The authors are grateful to Chemagnetics, Inc., for providing the sample-spinning system, and to the U.S. Geological Survey and U.S. Department of Energy (Laramie Energy Technology Center) for partial support of this research. 


\section{REFERENCES}

I. M. M. MARICQ AND J. S. WAUGH, J. Chem. Phys. 70, 3300 (1979).

2. J. S. WAugh, M. M. MARicQ, AND R. CANTOR, J. Magn. Reson. 29, 183 (1978).

3. E. LippmaA, M. Alla, AND T. Tuherm, "Proceedings, 19th Congress Ampere, Heidelberg, 1976," p. 113.

4. J. Herzfeld and A. Berger, J. Chem. Phys. 73, 6021 (1980).

5. W. P. Aue, D. J. Ruben, AND R. G. GRiffin, J. Magn. Reson. 43, 472 (1981).

6. Y. Yarim-AgaEV, P. N. TutunjIAN, AND J. S. WaUgh, J. Magn. Reson. 47, 51 (1982).

7. A. BAX, N. M. SzeverenYI, ANd G. E. MaCiel, J. Magn. Reson. 51, 400 (1983).

8. A. BAX, N. M. SzeverenYi, AND G. E. MaCiel, J. Magn. Reson. 52, 147 (1983).

9. E. O. StejsKal, J. SChaEfER, AND R. A. MCKay, J. Magn. Reson. 25, 569 (1977).

10. M. Mehring, "High Resolution NMR in Solids," pp. 40-43, Springer, New York, 1983.

11. G. Bodenhausen, R. Freeman, R. Niedermeyer, AND D. L. TurNer, J. Magn. Reson. 26, 133 (1977).

12. A. BAX, "Two-Dimensional Nuclear Magnetic Resonance in Liquids," pp. 26-30, Reidel, Boston, 1982.

13. Chemagnetics Inc., 208 N. Commerce Dr., Ft. Collins, Colo. 80524.

14. K. Nagayama, K. Wuthrich, P. BachmanN, and R. R. ErNSt, J. Magn. Reson. 31, 133 (1978). 